\title{
Rectangle Positioning Algorithm Simulation Based on Edge Detection and Hough Transform
}

\author{
Ping Xie ${ }^{*}$ and Jiang Zhou
}

North China Electric Power University, Beijing, 102206, China

\begin{abstract}
This paper proposes a new algorithm to extract the rectangle in images. It's goal is to meet the requirement of the truck's compartment positioning and assure that some works are done inside the truck's compartment. Firstly, the Canny operator is used to calculate the edge of the image, because it is sensitive to the image texture features. Secondly, the Canny edge is transformed with the Hough transform algorithm. The peak points in the Hough parameter space indicate the straight lines which may be the elements of the compartment. The sides of the rectangle are the straight lines which meet the conditions of the actual size of the compartment. Finally, the four vertices of the rectangle are solved in accordance with the geometric constraint of rectangle. The simulation experiment shows that the algorithm is effective.
\end{abstract}

Keywords: Edge detecting, Hough transform, rectangle positioning, simulation.

\section{INTRODUCTION}

Edge detection technology is increasingly been applied in various fields along with the development of computer vision and digital image processing, and its role is becoming more and more important. Coal is the important raw material in coal-fired power plants or steel mills. It plays a decisive role to in the quality of products. For small or medium-sized enterprises, the coal is carried into the factory mainly by trucks. While collecting the coal samples for further testing, the sampling process has been changed from manual sampling to mechanical sampling. But these sampling devices are unable to avoid the interference of man-made factors, because the sampling locations are decided by the operation personnel [1]. On this situation, an intelligent sampling control system is necessary to cut down the influence of the human factor, to improve the reliability of sampling system, and thus realize the goal of automatic sampling.

For the moment, the technologies suitable for compartment positioning mainly include infrared signals localization and ultrasonic positioning that is based on sensor, as well as digital image processing technology which extracts image edge information. The positioning technology based on the sensor is vulnerable to environmental factors (such as the coal dust or the moving objects), but the positioning technology based on image edge detection technology can overcome these problems.

References [2-6] have discussed image identification issues for straight line and rectangle. Although the compartments of the trucks are rectangle, but the location of the truck (in the detection area of the driveway) is not fixed

\footnotetext{
*Address correspondence to this author at the North China Electric Power University, Beijing, 102206, China; Tel: 0086-10-61772177; Fax: 0086-10-61772177; E-mail: pingxielaoshi@163.com
}

while waiting for sampling, and the size of the compartments vary from a truck to another. Consequently, the key for intelligent automatic sampling is how to obtain the locations of the compartments. Hence, those methods must be improved to apply to the truck's positioning.

For the truck's compartment positioning, [7] proposed a locating method based on background subtraction and image projection method; [8] presented an edge detection algorithm on fuzzy sets according to the requests of determining orientation of coal compartment. It is applicable to the edge detection of bigger objective area (compartment) of multilevels image. In this paper, according to the image detection characteristics of the truck's compartment filled with coal, a camera is set up to get the location of the truck image in real time, then the Canny operator for the image edge detection is used to calculate the edge of the truck image. The Canny edge image contains the front part of the truck. In order to assure that the sampling locations are inside the compartment, the rectangle area that indicates the truck compartment should be identified by the Hough transform.

\section{ALGORITHM PRINCIPLE}

\subsection{Detection Object Analysis}

As shown in Fig. (1), in order to get the location of the truck in real time, a camera is set up on the top middle of the sampling area. The truck image information is acquired from the dynamic video after the truck has stopped in the sampling area, then the rectangle area which indicates the truck's compartment could be identified by the digital image edge detection technology and Hough transform.

Roberts operator, Sobel operator, Prewitt operator, Log operator, Laplacian operator and Canny operator are familiar edge detection operators. Many scholars have made the analysis and comparison of among their respective advantages and disadvantages. The edge of the compartment 
has obvious characteristic due to the black coal, so the outline of the truck can be easily obtained with an edge detection algorithm. The Canny operator is used in this paper because it is sensitive to the image texture features.

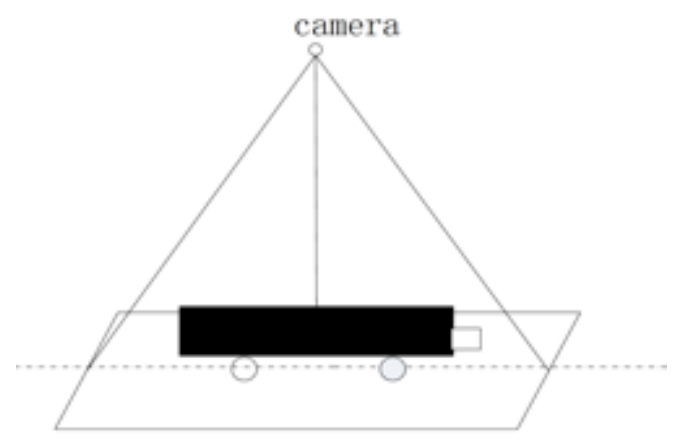

Fig. (1). The diagram for sampling area.

The length and width data of the truck can be retrieved directly from the truck's RFID tags identification system, and the sampling area is a one-way road. Consequently, the direction of the truck is fixed. We just need to identify the tail location of the truck, and then the whole poison of the truck compartment can be calculated with the rectangle geometry constraints.

\subsection{Canny Edge Detection}

Canny edge detection operator based on optimization algorithm was put forward by John F. Canny in 1986. The edge of an image is some areas where brightness changes significantly. The gray level in these areas is generally regarded as a step. That is in the gray scale of a small area, occurs dramatic changes. The Canny edge detection has three criteria [9].

\subsubsection{SNR Criterion}

The goal for SNR (Signal Noise Ratio) is to check out all pixels that are part of the edge and remove all pixels that do not belong to the edge. SNR can be expressed as (1):

$$
S N R=\frac{\left|\int_{-\infty}^{\infty} G(-x) f(x) d x\right|}{\delta \sqrt{\int_{-\infty}^{\infty} f^{2}(x) d x}}
$$

In (1), $f(x)$ is the filter impulse response in the boundary $[-\infty, \infty]$, is on behalf of the edge, and is the root mean square for Gauss noise.

\subsubsection{Criterion}

L (Localization accuracy) criterion shows that the edge pixels checked out must coincide with the actual edge as closely as possible. $\mathrm{L}$ can be expressed as (2):

$$
L=\frac{\left|\int_{-\infty}^{\infty} G^{\prime}(x) f^{\prime}(x) d x\right|}{\delta \sqrt{\int_{-\infty}^{\infty} f^{\prime 2}(x) d x}}
$$

In (2), is the first-order derivative of $G(x)$, and $f(x)$ is the first-order derivative of $f(x)$.

\subsubsection{Single Edge Response Criteria}

A single edge has only one response, and the false edge response should be restrained as much as possible. It can be expressed as D (the average Distance of the false edge) (3):

$$
D=\pi \sqrt{\frac{\int_{-\infty}^{\infty} f^{\prime 2}(x) d x}{\int_{-\infty}^{\infty} f^{\prime}(x) d x}}
$$

For the three criteria, SNR reflects the detection rate of an edge detection operator based on the real edge. The higher the SNR, the more likely the detection result contains the real edges and cuts off the false edges. $\mathrm{L}$ reflects the mathematical expectation between the detection result and the inverse of the actual edge. The Lower the L, the higher the localization accuracy. D represents the average distance of the false edges by the convolution between the random noise and the detection function. The higher the $\mathrm{D}$, the lower possibility the false edges are.

In this paper, Canny operator assumes that average distance $\mathrm{D}$ is a constant, and considers the optimal product of SNR and L which is calculated in limited times as Canny detection function, and then it is expressed as the firstorder derivative of Gauss function. If the Gauss function was $G(x)=e^{-\alpha^{2} x^{2} / 2}$, the Canny operator is as follows:

$C a(x)=x e^{-\alpha^{2} x^{2} / 2}$

Canny operator has a higher fidelity on the edge of original image, and also has better edge location accuracy. Hence the Canny operator is adopted.

\subsection{Rectangle Detection Principle of Hough Transform}

Hough transform is a kind of regional boundary shape description method. It is usually used to detect the straight line, circle or ellipse. This method has higher reliability, and can output the ideal result even under the condition of noise, deformation or some loss of image.

The basic idea to detect straight lines by Hough transform is the duality principle of points and lines. In other words, the points and sine curves are dual. On the one hand, any point in a straight line can correspond to those sine curves in the Hough transform space which intersect at the same point; on the other hand, the sine curves in the Hough transform space which intersect at the same point also can correspond to a straight line. So the Hough transform changes the problems of detecting straight lines in the image space into detecting points in the Hough transform space. A straight line in the image space usually is expressed as an equation that has a constant slope of $\mathrm{k}$, such as $y=k x+b$. In this way, the size of the accumulator array could be very large when the slope of $\mathrm{k}$ is infinity, and then the storage space required becomes larger. In order to solve this problem, the straight lines are expressed as polar equation: 
$\rho=x \cos \theta+y \sin \theta$

According to (5), a straight line in the original image space is mapped to a point in the Hough transform space, as shown in Fig. (2).

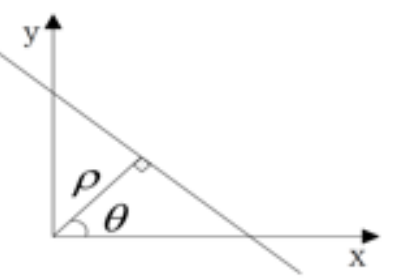

Image space

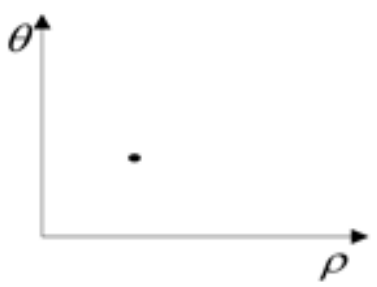

Hough transform space
Fig. (2). A line in the image space is mapped to a point in the Hough transform space.

Every point of the straight line in the image space is mapped to the same point in the Hough transform space because they have the same slope and intercept. As a result, the straight line in the image space becomes a rallying point in the Hough transform space after mapping. The length of the straight line segment determines the total value of the rallying point. In this way, the problem of detecting straight lines is replaced with finding peak points in Hough transform space.

Consider the rectangle in Fig. (3). 0 is the coordinate origin. $P_{1}\left(x_{1}, y_{1}\right) P_{2}\left(x_{2}, y_{2}\right), P_{3}\left(x_{3}, y_{3}\right)$ and $P_{4}\left(x_{4}, y_{4}\right)$ are the four vertices of the rectangle. The length of sides are a and $b$.

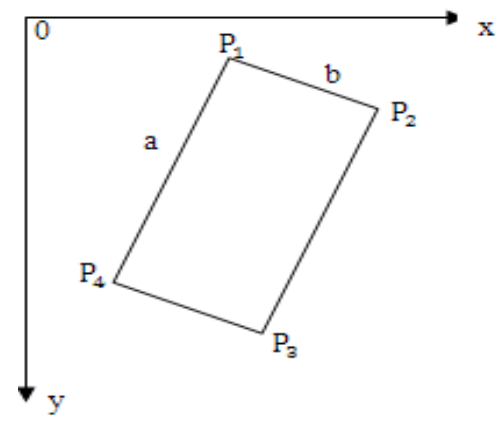

Fig. (3). A rectangle in the image.

As shown in Fig. (4), the rectangle is changed after Hough transform. There are 4 peak points: $C_{1}\left(\rho_{1}, \theta_{1}\right)$, $C_{2}\left(\rho_{2}, \theta_{2}\right), C_{3}\left(\rho_{3}, \theta_{3}\right)$ and $C_{4}\left(\rho_{4}, \theta_{4}\right)$. They are respectively corresponding to the four sides of the rectangle: $P_{2} P_{3}$, $P_{4} P_{1}, P_{3} P_{4}$ and $P_{1} P_{2}$.

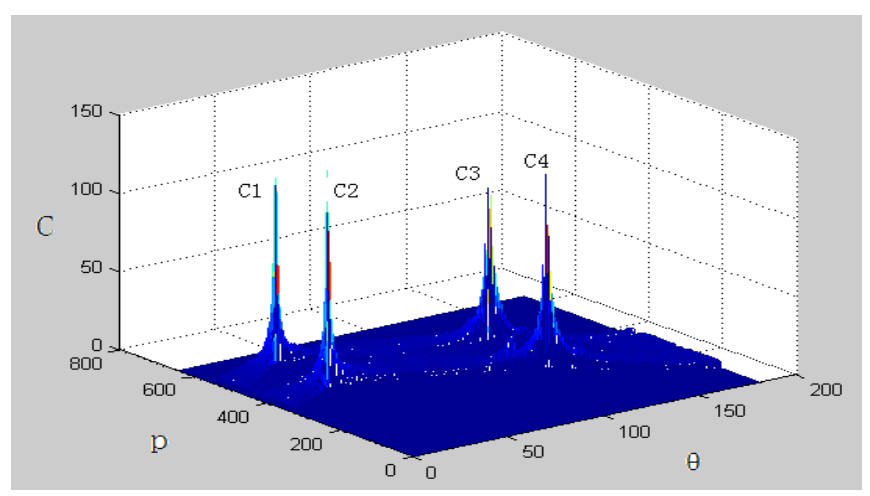

Fig. (4). The rectangle in the Hough transform space.
The four peak points satisfy the following conditions:

They are conjugates. $C_{1}$ and $C_{2}$ are a pair that meets the condition of $\theta_{1}=\theta_{2}$, and $C_{3}$ and $C_{4}$ are another pair that meets the condition of $\theta_{3}=\theta_{4}$.

The abscissa gap between two pairs of peak points is $90^{\circ}$, that means $\left|\theta_{1}-\theta_{3}\right|=90^{\circ}$ or $\left|\theta_{2}-\theta_{4}\right|=90^{\circ}$

Because the length of the rectangle's opposite sides are equal, the same pair of peak points' total value are also equivalent. That means $C_{1}=C_{2}$ and $C_{3}=C_{4}$.

The distance between the same pair of peak points is equal to the length of the rectangle's side, which means $\left|\rho_{1}-\rho_{2}\right|=b$ and $\left|\rho_{3}-\rho_{4}\right|=a$.

\section{RECTANGLE COMPARTMENT POSITIONING ALGORITHM}

\subsection{Algorithm Idea}

As long as the tail part of the compartment is identified, in other words, a pair of vertices and three sides of the rectangle are found out, the rectangle area of the whole compartment excluding the front part can be calculated. The reason is that the length and width of the compartment has been given and the truck enters the sampling area only in one way. Accordingly, we just need to process half of the truck image. The algorithm is as follows:

Step 1: Obtain edge information of the truck after dealing with the truck image with Canny edge detection operator.

Step 2: Cut out half of the Canny edge image to perform rectangle feature extraction with the windowed Hough transform technique.

Step 3: Extract all straight lines in the window which would belong to the tail edge of the truck in Hough transform space.

Step 4: Eliminate those straight lines which do not meet specific conditions. That means the straight lines which are not part of the truck would be removed.

Step 5: Find out a pair of vertices (that is, two intersection points of three straight lines) which indicates the tail of the truck's rectangle region.

Step 6: Use the rectangle geometric constraints to calculate the other two vertices.

Since the four vertices of the rectangle have been identified, the compartment area also can be confirmed. In the following subsections, some of these steps will be described in detail.

\subsection{Straight Line Extraction Algorithm}

In order to find out the straight lines, the Canny edge image should be mapped into the Hough transform space by (5). The Hough transform is used for the whole image, so the range of $\rho$ depends on the size of the image and is related to the diagonal length of the image, the range of which should be $0 \sim 180^{\circ}$. The array $C(\rho, \theta)$ is used to cumulate the number of those pixels in the Canny image that meet the straight line equation. 

follows:

The specific process for straight lines extraction is as

Step 1: For each edge pixels $(x, y)$ in Canny image, each $\theta$ can be used to calculate a corresponding value $\rho$. The $\theta$ may take over any possible value in the range of $0 \sim 180^{\circ}$, each $\rho$ and $\theta$ constitute the only one straight line in the Canny image. The values of array $C(\rho, \theta)$ are accumulated according to the value of $\rho$ and $\theta$, so we can get the numbers of pixels meeting the same parameters of $\rho$ and $\theta$.

Step 2: Find out the peak points in the array $C(\rho, \theta)$, because each peak point is corresponding to a straight line. Method for finding the peak point is to extract all points which meet the threshold condition $C(\rho, \theta) \geq T_{c}$, and threshold $T_{c}$ is associated with the image size.

\subsection{Vertex Extraction Algorithm}

We have known that $C_{1}\left(\rho_{1}, \theta_{1}\right), C_{2}\left(\rho_{2}, \theta_{2}\right), \cdots$, $C_{m}\left(\rho_{m}, \theta_{m}\right)$ are $m$ peak points extracted from array $C(\rho, \theta)$, the goal of this step is to find out a pair of vertices (that is, two intersection points of three straight lines) which indicates the tail of the truck's rectangle region.

Firstly, we must find out a pair of parallel sides belonging it to the compartment by scanning each peak point. It means two peak points which meet the following condition (6) make a pair.

$\left|\theta_{i}-\theta_{j}\right|<T_{\theta}$ and ||$\rho_{i}-\rho_{j}|-W|<T_{w}$

In (6), $T_{\theta}$ is the angle threshold, $\mathrm{W}$ is the actual width of the compartment, $T_{w}$ is the width deviation threshold.

Among those peak points which are not paired successfully, the third side of the compartment $C_{k}\left(\rho_{k}, \theta_{k}\right)$ is the one which satisfies the following conditions (7).

||$\theta_{k}-\frac{\theta_{i}+\theta_{j}}{2}\left|-90^{\circ}\right|<T_{\alpha}$

In (7), $T_{\alpha}$ is the angle threshold.

Now the three peak points we found out just represent the three sides of the tail compartment.

Finally, the rest of the job is to extract the two vertices of the tail compartment.

Assuming the three peak points we find out are $C_{1}\left(\rho_{1}, \theta_{1}\right), C_{2}\left(\rho_{2}, \theta_{2}\right)$ and $C_{4}\left(\rho_{4}, \theta_{4}\right)$, as shown in Fig. (4). As an example, we deal with $C_{1}$ and $C_{4}$ to illustrate how to get a vertex. The intersection point of the two straight lines is exactly the vertex.

We can get (8) by feeding the parameters $C_{1}\left(\rho_{1}, \theta_{1}\right)$ into (5) and get (9) by feeding the parameters $C_{4}\left(\rho_{4}, \theta_{4}\right)$ into (5).

$$
\begin{aligned}
& \rho_{1}=x \cos \theta_{1}+y \sin \theta_{1} \\
& \rho_{4}=x \cos \theta_{4}+y \sin \theta_{4}
\end{aligned}
$$

The intersection point of the two straight lines can be calculated by (8) and (9), and the intersection point is corresponds to the vertex $P_{2}\left(x_{2}, y_{2}\right)$.

Similarly we can calculate the intersection point of $C_{2}$ and $C_{4}$ which corresponds to the vertex $P_{1}\left(x_{1}, y_{1}\right)$. Thus we obtained a pair of vertices and three sides of the compartment.

\subsection{Solving Rectangle Vertices}

The problem of solving rectangle vertices can be transformed into solving the location of the vertices $P_{3}\left(x_{3}, y_{3}\right)$ and $P_{4}\left(x_{4}, y_{4}\right)$ in Fig. (3), because we obtained the location of vertices $P_{1}\left(x_{1}, y_{1}\right), P_{2}\left(x_{2}, y_{2}\right)$, three edges, and the length of a and $\mathrm{b}$ as shown in Fig. (3).

The three peak points of the rectangle are $C_{1}\left(\rho_{1}, \theta_{1}\right)$, $C_{2}\left(\rho_{2}, \theta_{2}\right)$ and $C_{4}\left(\rho_{4}, \theta_{4}\right)$. According to the relationship among the four Hough peak points described in Section 2, the last peak point $C_{3}\left(\rho_{3}, \theta_{3}\right)$ should meet the condition of $\left|\rho_{3}-\rho_{4}\right|=a$ and $\theta_{3}=\theta_{4}$. The vertex $P_{3}\left(x_{3}, y_{3}\right)$ is exactly the intersection point of $C_{1}$ and $C_{3}$, and the vertex $P_{4}\left(x_{4}, y_{4}\right)$ is exactly the intersection point of $C_{2}$ and $C_{3}$. Hence, refer to the (5) we can work on them.

\section{SIMULATION EXPERIMENT}

Firstly, the Canny operator is used to detect the edge of the original image. The original image is shown in Fig. (5), whereas the Canny edge image is shown in Fig. (6). The front part of the truck is included in the Canny edge.

Secondly, cut out half of the Canny edge image to perform rectangle feature extraction with the windowed Hough transform algorithm. Fig. (7) is the image cut out to detect the tail part of the compartment.

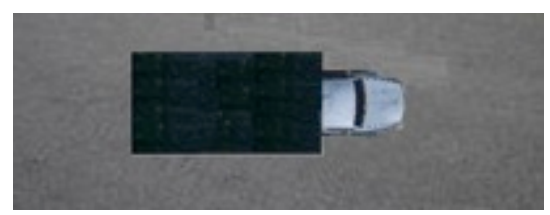

Fig. (5). The original image.

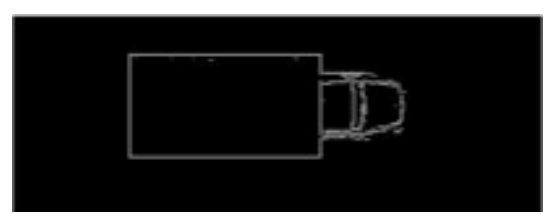

Fig. (6). The Canny edge image.

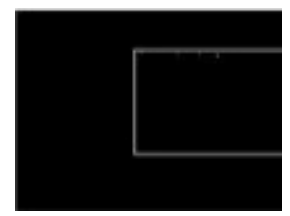

Fig. (7). The tail part of the Canny edge image.

\subsection{Extracting the Straight Lines}

Firstly, the Canny edge image should be mapped into the Hough transform space by (5). The range of $\rho$ has been defined as 2 times of the diagonal length of image, the range of $\theta$ was supposed to be $0 \sim 180^{\circ}$, but we can narrow it down 
and reduce the time for Hough transform. The reason is because the sampling area is a one-way road, the direction of the truck is fixed, so it will be limited to a certain range. In this paper, the range of $\theta$ is set to $0 \sim 20^{\circ}, 70 \sim 110^{\circ}$ and $160 \sim 180^{\circ}$, which means the inclination of the compartment is not more than $20^{\circ}$.

After that, it is time for finding out the peak points of Hough transform and extracting all points that meet the threshold condition $C(\rho, \theta) \geq T_{c}$. Here $T_{c}$ is set to $0.2 \mathrm{~W}$, that is to say, $T_{c}=0.2 \mathrm{~W}$. W is the actual width of the rectangle compartment taken from the truck RFID tags identification system. Each peak point corresponds to a straight line. The tail part of the compartment includes 3 lines, and the 3 extracted straight lines are shown in Fig. (8).

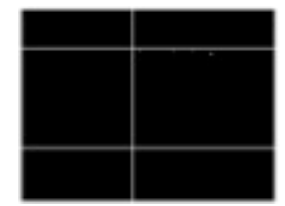

Fig. (8). The straight lines extracted from the Hough transform space.

\subsection{Extracting the Vertices}

Firstly, scan each peak point extracted to find out a pair of parallel edges which satisfies (6). In this paper, edges are set to 3 .

Secondly, the third edge of the compartment is found out among those peak points which are not paired successfully. The third edge is the peak point which satisfies (7).

According to (8) and (9), we can calculate two intersection points related with the 3 straight lines shown in Fig. (8).

\subsection{Determining the Rectangle Region}

According to the relationship among the four Hough peak points described in section 2 , the fourth characteristic straight line of the compartment can be obtained. So we can get the other two vertices by finding out the intersection points among the fourth straight line and the two parallel lines. As shown in Fig. (9), the rectangle region of the compartment is the area marked by connecting the four vertices.

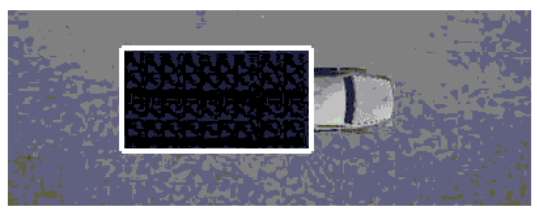

Fig. (9). The rectangle indicates the location of the compartment.

\section{CONCLUSION}

In this paper, a new algorithm has been proposed to position the truck full of coal. In the power plants and steel mills whose main material is coal, the sampling control device must know the sampling locations which are inside the compartment. The algorithm is based on the digital image processing technology. It is achieved by Canny edge and Hough transform. Firstly, the Canny operator is used to calculate the edge of the image, because it is sensitive to the image texture features. Secondly, the Canny edge is transformed with the Hough transform algorithm. The peak points in the Hough parameter space indicate the straight lines which may be the elements of the compartment. The actual size of the compartment is introduced into the calculating, hence, the positioning accuracy of the truck can be increased and it is important for the coal sampling automation.

\section{CONFLICT OF INTEREST}

The authors confirm that this article content has no conflict of interest.

\section{ACKNOWLEDGEMENTS}

Declared none.

\section{REFERENCES}

[1] S. Y. Xie, Z. Y. Zhao, Z. Q. Shang, and Z.W. Zhao, "Study on belt samplers for truck-coal in a coal-fired power plant," Electric Power, vol. 45, pp. 39-42, 2012.

[2] S. S. Bao, and L. Yang, "Algorithm for detecting vertical and horizontal lines in real-time image processing," Journal of Hefei University of Technology, vol. 26, pp. 550-552, 2003.

[3] Q. Q. Wang, M. Y. Cao, N. L. Sun, and D. Fan, "Accurate localization algorithm for rectangle mark based on spatial interpolation technology and combined detection method," The 2008 International Congress on Image and Signal Processing, Sanya: China, 2008, pp. 79-84.

[4] Z. Yuan, and Q. Z. Zhu, "Rectangle detection by the chain-code tracing," The 2nd International Conference on Electrical and Control Engineering, Yichang: China, 2011, pp. 759-762.

[5] T. T. Nguyen, X. D. Pham, and J. W. Jeon, "Rectangular object tracking based on standard hough transform," The 2008 IEEE International Conference on Robotics and Biomimetics, Guilin: China, 2008, pp. 2098-2103.

[6] C. R. Jung, and R. Schramm, "Rectangle detection based on a windowed hough transform," The 2004 IEEE XVII Brazilian Symposium on Computer Graphics and Image Processing, Curitiba: Brazil, 2004, pp. 113-120.

[7] C. Zhou, "Car position locating method based on image processing," Computer systems \& applications, vol. 20, pp. 219-221, 2011.

[8] Q. X. Yang, and H. Y. Wu, "New fuzzy EDA and its application in determining orientation of coal compartment," Computer Engineering and Design, vol. 28, pp. 4187-4189, 2007.

[9] J. Canny, "A computational approach to edge detection," IEEE Transactions on Pattern Analysis and Machine Intelligence, vol. 8, pp. 679-697, 1986. 